\title{
La Evaluación Educativa: aproximación a un caso de $1^{\circ}$ de la ESO The Educational Evaluation: approach to a case of 1 st compulsory secondary education
}

\author{
Ma Eugenia Azambuya Bouzón ${ }^{1}$
}

Recibido: 19 de noviembre de 2019 Aceptado: 17 de diciembre de 2019 Publicado: 31 de enero de 2020

To cite this article: Azambuya, M ${ }^{\mathrm{a}}$ E. (2020). La Evaluación Educativa: aproximación a un caso de $1^{\circ}$ de la ESO. Márgenes, Revista de Educación de la Universidad de Málaga, 1 (1), 240-262

DOI: http://dx.doi.org/10.24310/mgnmar.v1i1.7153

\author{
${ }^{1}$ Ma Eugenia Azambuya Bouzón \\ Universidad Católica del Uruguay \\ meugenia.azambuya@gmail.com
}

\begin{abstract}
RESUMEN
El artículo que aquí se presenta expone el acercamiento a un caso de primero de la ESO de un Instituto de Educación Secundaria de la provincia de Málaga. El objetivo del estudio ha sido conocer y comprender las concepciones que poseen docentes y estudiantes de dicho curso sobre la evaluación educativa, así como la forma en que la misma se materializa, y las diferencias que se establecen respecto a la calificación. Para ello, se ha partido de una observación inicial, se realizaron entrevistas a los agentes educativos mencionados así como a la orientadora del instituto, y se analizaron ciertos documentos de interés. De acuerdo a la información recopilada y contrastada, parecería existir confusión respecto al significado y a las funciones de la evaluación. Esta última se suele fusionar con la calificación, lo cual repercute inevitablemente en la metodología de enseñanza de los docentes y por ende, en el proceso de aprendizaje del alumnado.
\end{abstract}

Palabras clave: profesorado; alumnado; evaluación educativa; calificación

\begin{abstract}
The article exposes an approach to a case of first year ESO in a Secondary Education Institute of Málaga province. The goal was to approach and understand the conceptions that teachers and students from this course have concerning the educational evaluation, so as the way in which it is performed, and the differences established regarding the qualifications. To achieve this, the process began with an initial observation, interviews with the educational agents mentioned, as well as the guidance of the institute were conducted, and certain documents of interest were reviewed. According to the gathered and contrasted information, it seems to be confusion regarding the meaning and functions of the evaluation. The latter is usually merged with the measurement, which inevitably affects the teaching methodology, and therefore, the student learning process.
\end{abstract}

Keywords: teachers, students, educational evaluation, measurement 


\section{E S T U D I A N T E S}

\section{INTRODUCCIÓN: HABLEMOS SOBRE LA EVALUACIÓN EDUCATIVA}

Según Parcerisa y Zabala (1996), el papel que se le otorga a la evaluación y la forma en la cual la misma es llevada a cabo pueden determinar el proceso educativo, llegando a condicionar a los objetivos propuestos y entrando en algunas oportunidades, en evidente contradicción con los mismos. A su vez, la función que cumpla la evaluación incidirá indefectiblemente en la metodología de quien enseña, así como en el clima de clase y el proceso de aprendizaje de los estudiantes.

Ahora bien, a pesar de ser un concepto tan evocado y relevante para el sistema educativo, desde siempre se han producido confusiones con respecto al mismo. Al decir de Rubio, Castillejo, Sánchez y López (1992) así como de Alcaraz (2015c), un problema que se suele presentar es que se confunde la evaluación con la calificación, lo cual repercute claramente tanto en los docentes como en los estudiantes, desvirtuándose así las funciones de un proceso que en su estado puro persigue ante todo, la comprensión y la mejora del aprendizaje.

La Orden de 14 de julio de 2016, por la que se desarrolla el currículo correspondiente a la Educación Secundaria Obligatoria en la Comunidad Autónoma de Andalucía, se regulan determinados aspectos de la atención a la diversidad y se establece la ordenación de la evaluación del proceso de aprendizaje del alumnado, establece en el artículo 14 una serie de consideraciones fundamentales respecto a cómo debe ser concebida la evaluación en dicha etapa del sistema educativo, y qué características debe poseer su puesta en práctica. Entre dichas consideraciones, se encuentran las siguientes:

a) Que la evaluación será continua, formativa, integradora y diferenciada según las distintas materias.

b) Que teniendo en cuenta su carácter formativo y su consideración como instrumento para la mejora, el profesorado deberá evaluar tanto los aprendizajes del alumnado como los procesos de enseñanza y su propia práctica docente.

c) Que la evaluación del proceso de aprendizaje deberá ser integradora, pero el profesorado podrá realizarla de manera diferenciada en función de la asignatura.

En relación a la continuidad, que la evaluación se proclame continua implica que se encuentra inmersa en el proceso de enseñanza-aprendizaje, no como un apéndice sino como parte misma y fundamental de dicho proceso, persiguiendo el fin de detectar posibles dificultades, analizar sus causas, y en consecuencia, adaptar las prácticas educativas (Morey, 2001).

Cuando decimos que además la evaluación debe ser formativa, nos referimos a ella como actividad crítica de aprendizaje, ya que poniéndola en práctica se adquieren nuevos conocimientos (Álvarez Méndez, 1993). El docente aprende para conocer y mejorar su práctica, mientras el alumno se apropia de la información contrastada 


\section{E S T U D I A N T E S}

que le ofrece el anterior, que ha de ser crítica y argumentada pero en ningún caso descalificadora o penalizadora (Álvarez Méndez, 2008). Si atendemos precisamente a este punto, se puede detectar que el mismo entra en evidente contradicción con la asignación de la calificación.

En otras palabras, Popham (2013) habla de un proceso planificado en el que tanto docentes como alumnos utilizan la información que se desprende del mismo para realizar ajustes en lo que están haciendo. A su vez, para que dicho proceso sea efectivamente formativo, los ajustes deben realizarse lo antes posible, es decir, cuando aún se disponga de tiempo para la enseñanza y el aprendizaje (Álvarez Méndez, 2008; Popham, 2013; Santos Guerra, 2014).

Continuando con la condición integradora, la misma hace alusión a la necesidad contemplar las capacidades generales que se establecen para la etapa educativa, de acuerdo a los objetivos de las diversas áreas y asignaturas (Morey, 2001). Nos referimos con esto a una evaluación que se ajuste a las metas que persigue la educación, es decir, a las habilidades que se espera que desarrollen tanto los estudiantes como los docentes.

Por último, en su carácter diferencial, la evaluación puede -y debería- adoptar características particulares dependiendo del contexto, el profesorado, los estudiantes, y la asignatura de la cual se trate.

En definitiva, tal como se explicita en la Orden así como en las diversas definiciones existentes de la evaluación formativa (Álvarez Méndez, 2008; Fernández Navas, Fernández Millán y Hamido Mohamed, 2011; Popham, 2013; Santos Guerra, 2014), se deben evaluar todos los elementos que hacen al proceso de enseñanza aprendizaje.

A su vez, es necesario que la evaluación sea transparente, creíble, con coherencia epistemológica y cohesión práctica, pertinente, aplicable y legítima, y para ello, debe estar al servicio de quien enseña y de quien aprende. Esto último constituye un componente fundamental que todos los agentes educativos deberían comprender, ya que conforma la base necesaria para poder cambiar la relación entre alumnado y profesorado, y que la misma pase a sustentarse en la cooperación, participación y corresponsabilidad.

\section{EL PROPÓSITO DE ESTE ESTUDIO}

La intención de este trabajo ha sido indagar, conocer y comprender las concepciones que tanto alumnos como docentes de $1^{\circ}$ de la ESO de un instituto malagueño poseen sobre la evaluación educativa. En el caso de los docentes, descubrir y comprender también como dichas concepciones se materializan en sus prácticas, y en el caso de los alumnos, indagar sobre la forma en que se sienten al ser evaluados. 


\section{E S T U D I A N T E S}

Las preguntas que han orientado el proceso se presentan en la siguiente tabla:

\begin{tabular}{|ll|}
\hline Sujetos & Preguntas a responder \\
\hline & 1) ¿Qué concepciones poseen los docentes de $1^{\circ}$ de la ESO del instituto acerca de \\
Docentes & 2) ¿Cómo llevan a cabo dichos docentes la evaluación? \\
& 3) ¿Experimentan estos docentes dificultades a la hora de realizar la evaluación? \\
& 4) ¿Perciben estos docentes las diferencias entre la evaluación y la calificación? \\
\hline Alumnado & 1) ¿Qué concepciones manejan los estudiantes de $1^{\circ}$ de la ESO del instituto \\
& 2) ¿Cómo viven dichos estudiantes el hecho de ser evaluados? \\
\hline $\begin{array}{l}\text { Centro } \\
\text { educativo }\end{array}$ & 3) ¿Perciben estos estudiantes la diferencia entre evaluación y calificación? \\
\hline
\end{tabular}

Tabla 1: Las preguntas a responder

\section{METODOLOGÍA}

Para intentar responder a las interrogantes propuestas, se decidió realizar una aproximación a la investigación cualitativa, y particularmente al estudio de caso. Según McMillan y Schumacher (2005) la investigación cualitativa permite la recopilación de datos en situaciones reales, mediante la interacción con personas seleccionadas en su propio entorno. En consonancia con ello, Flyvbjerg (2006) agrega que la forma más avanzada de comprensión de los fenómenos se logra cuando los investigadores se ubican dentro el contexto de estudio. Solo así se pueden comprender en profundidad los puntos de vista y comportamientos de los sujetos.

En definitiva, este tipo de investigación se ocupa concretamente de comprender los fenómenos sociales desde la visión de los participantes, es decir, a través de sus ideas, sentimientos, creencias, pensamientos y conductas (Pasek y Mejía, 2017; Stake, 2010). Al decir de Osses, Sánchez e Ibáñez (2006) las bases epistemológicas que posee dicho enfoque estriban en la singularidad de los fenómenos sociales, que precisan ser estudiados mediante metodologías también singulares, en un proceso de interrelación mutua entre objeto y sujeto, donde importa más la peculiaridad que la generalización.

En cuanto al estudio de caso, Muñoz y Muñoz (2000) argumentan que su objetivo es comprender la particularidad del caso en cuestión, en el intento de analizar cómo funcionan los componentes que lo conforman y las relaciones entre ellos para formar un todo. Schutt (2011) agrega que el caso puede ser una persona, un grupo, una organización formal, una comunidad u otras colectividades. Lo importante es poder reflejar con claridad el punto de vista de los actores sociales estudiados y para ello se utilizan 


\section{E S T U D I A N T E S}

múltiples fuentes de datos (como la observación, las entrevistas, el análisis de documentos, etcétera) y se interactúa con el contexto durante un largo período de tiempo.

En este trabajo, la elección del caso persigue un interés intrínseco, ya que el mismo viene dado y se quiere aprender concretamente sobre sus características (Stake, 2010). Sin embargo, dicho interés se complementa con el instrumental, ya que también se busca profundizar y comprender sobre el tema en general.

\subsection{Un acercamiento al instituto}

El centro educativo en cuestión, al que llamaremos "A", atiende a una población compuesta por aproximadamente 600 estudiantes de diversa procedencia, la cual asiste al instituto diariamente entre las 8.15 y las 14.45 horas. En cuanto al equipo docente, el mismo está conformado 60 profesores.

El contexto en el cual se enclava es de nivel socioeconómico medio-bajo, y el alumnado, proviene de diversos niveles socioculturales, existiendo una gran cantidad de estudiantes extranjeros. Es por dichas características que el centro educativo está catalogado como de compensatoria. Según la Orden del 22 de julio de 1999, por la que se regulan las actuaciones de compensación educativa, las actuaciones de dichas instituciones estarían destinadas a garantizar la escolarización asegurando la igualdad de oportunidades para todo el alumnado, teniendo en cuenta la situación inicial de desventaja social. Para cumplir con ello, se aspira a lograr la participación de los diferentes sectores de la comunidad educativa en las actuaciones que se lleven a cabo y se busca establecer canales efectivos de comunicación para garantizar la retroalimentación de la información y la participación de las familias.

Esto último resulta un punto débil en este instituto particular, ya que las familias se encuentran escasamente involucradas con el centro y no se ha logrado establecer una comunicación fluida con las mismas hasta el momento. No se cuenta con Asociación de Madres y Padres de Alumnos (AMPA) y al decir de la orientadora, la asistencia de las familias a las actividades que buscan fortalecer su participación dentro del centro es prácticamente nula.

En cuanto a las enseñanzas que se imparten, existen dos grupos de $1^{\circ}, 2^{\circ}$ y $3^{\circ}$ de Educación Secundaria Obligatoria. En el caso de $4^{\circ}$ existe un único grupo. A su vez, para el caso de $2^{\circ}$ y $3^{\circ}$ de la ESO se cuenta con un grupo del Programa para la Mejora del Rendimiento y del Aprendizaje (PMAR). En lo que refiere a Bachillerato se dictan las opciones de Ciencias, y de Humanidades y Ciencias Sociales. Por último, se ofrecen también diversos ciclos formativos.

\subsection{La recogida y organización de la información}

En primer lugar, es de destacar que para la recopilación de información se ha otorgado importancia a la triangulación, la cual busca aportar precisión a los datos, para aumentar el crédito de su interpretación (Stake, 2010). Denzin (1970, cit. en 


\section{E S T U D I A N T E S}

Maxwell, 2012) expone que la misma se basa en la integración de diversos métodos y fuentes de información, en vías de reducir el riesgo de que las conclusiones reflejen únicamente los sesgos o limitaciones de un método específico. Respecto de ello, Tracy (2010, p. 843) afirma que "los múltiples tipos de datos, los puntos de vista de los investigadores, los marcos teóricos y los métodos de análisis permiten explorar diferentes facetas de los problemas, aumentan el alcance, profundizan la comprensión, y fomentan la interpretación consistente."

En este caso particular se acudió a distintas fuentes de datos y se intentó llevar a cabo la triangulación metodológica (Stake, 2010), aunque surgieron ciertos inconvenientes que debilitaron su puesta en práctica. Las técnicas elaboradas para la recolección de datos así como las fuentes a las que se acudió se describen a continuación.

Se utilizó en primera instancia la observación de una sesión de evaluación de $1^{\circ}$ de la ESO. Según Stake (2010), la observación conduce a una mejor comprensión del caso. La particularidad de la que aquí se ha realizado es que la misma fue el "motor" del trabajo en cuestión, y ha sido no estructurada (Campos y Lule, 2012), ya que el foco no estuvo puesto sobre categorías o indicadores, sino sobre la situación en general. A su vez, la misma fue de tipo no participante ya que las conductas e interacciones ocurridas se dieron como lo harían sin la presencia del observador (Adler y Adler, 1998, cit. en Flick, 2012).

En segundo lugar, se ha diseñado un cuestionario, el cual constituye un tipo de encuesta, que se utiliza para "conocer las actitudes, las creencias, los valores, las características demográficas, los comportamientos, las opiniones, los hábitos, los deseos y las ideas de las personas" (McMillan y Schumacher, 2005, p. 292), además de otros tipos de información. En este caso, se creó en primer lugar una breve encuesta sociodemográfica para recopilar ciertos datos personales que serían útiles para el análisis de la información obtenida. Luego, se generó un cuestionario basado en bibliografía reciente sobre la evaluación educativa (Alcaraz, 2015a; Álvarez Méndez, 2008; Hortiguela, Pérez-Pueyo y González-Calvo, 2019; Santos Guerra, 2014), tomándose como ejemplo algunos ítems de la herramienta elaborada por Alfageme, Miralles y Monteagudo (2011). Su elaboración se llevó a cabo en vías de indagar y comprender la diferenciación que realizan los docentes entre los conceptos de evaluación y calificación y descubrir los procedimientos de evaluación que estos llevan a cabo en el instituto, los instrumentos que más utilizan y el feedback que les ofrecen a los estudiantes.

Por otra parte, se han elaborado entrevistas cualitativas, basadas en guiones con preguntas abiertas y semiabiertas, tanto para los docentes como para la orientadora y los estudiantes del instituto. Se eligió dicho método ya que permite poseer un protocolo de guía para la recolección de información, lo cual facilita la sistematización, a la vez que permite apertura en las respuestas y otorga flexibilidad a la entrevista (Valles, 2007, cit. en Lagoa, 2014). Respecto al tipo de preguntas, las mismas 


\section{E S T U D I A N T E S}

apuntan en este caso a indagar sobre comportamientos, opiniones, conocimientos y sentimientos (McMillan y Schumacher, 2005) de los entrevistados.

En último lugar, se ha recurrido a la revisión y análisis de documentos. Stake (2010) destaca que casi todos los estudios requieren la examinación de documentos y argumenta que ante la revisión de los mismos se debe tener la mente organizada a la vez que abierta a pistas inesperadas. Concretamente en este trabajo se ha recurrido a la revisión del proyecto educativo de centro y del modelo del guion para el desarrollo de las sesiones de evaluación.

Ahora bien, resulta trascendente aclarar que si bien la observación, las entrevistas y la revisión de los documentos pudieron realizarse de acuerdo a lo planificado, los cuestionarios no pudieron ser tenidos en cuenta para el trabajo, ya que no recibieron respuesta por parte de los docentes. En total, fueron entrevistados cinco profesores y catorce estudiantes de $1^{\circ}$ de la ESO, además de la orientadora del instituto.

Una vez recopilados los datos, se procedió a la codificación, categorización y triangulación de los mismos. Al decir de Maxwell (2012), el objetivo de la codificación es fracturar los datos y organizarlos en categorías, en vías de facilitar su comparación y comprensión. Así, la categorización hace más accesible el entendimiento general del fenómeno de estudio y la generación de nuevas ideas y conexiones basadas en los datos presentados. Esto último se hace posible al combinar la categorización con la triangulación (Stake, 2010).

Las categorías elaboradas para la interpretación de los datos se muestran en la siguiente tabla.

\begin{tabular}{ll} 
Categorías & Subcategorías \\
\hline \multirow{2}{*}{ La evaluación educativa } & Definición \\
& Funciones \\
& Nivel de dificultad percibido \\
\hline \multirow{2}{*}{ El procedimiento de evaluación } & Transparencia de criterios \\
& Instrumentos puestos en juego \\
\hline \multirow{2}{*}{ La calificación } & Feedback sobre el proceso \\
\hline & Definición \\
& Reparto de su peso \\
\hline
\end{tabular}

Tabla 2: Categorización para el análisis de los datos

\section{PRESENTACIÓN Y ANÁLISIS DE LOS DATOS}

Antes de presentar los datos recogidos, es necesario expresar que los mismos poseen sentido únicamente en relación al contexto particular en el que han sido obtenidos. Si bien se ha pretendido llegar a una mayor comprensión sobre la eva- 


\section{E S T U D I A N T E S}

luación educativa en general, la misma se encuentra estrechamente ligada al caso en cuestión y por lo tanto, no se aspira de ningún modo a la generalización.

\subsection{La evaluación educativa}

Tanto en la Ley Orgánica 8/2013, para la mejora de la calidad educativa, como en el Decreto 111/2016 y en la Orden del 14 de julio de 2016, se afirma que la evaluación debe ser continua, formativa e integradora. Lo mismo se estipula en el Proyecto de Centro (2018) del instituto, en el cual se explicitan dichas características. Ahora bien, las definiciones aportadas por los docentes ante la pregunta "¿qué significa para ti evaluar?" no parecen seguir la misma perspectiva. Las mismas (que pueden ser observadas en la Tabla 3) dan cuenta, entre otros factores, de que la evaluación según su parecer debe realizarse únicamente sobre el alumnado. De hecho, los estudiantes reconocen ser el único objeto de la evaluación, y ante la misma pregunta realizada a los docentes, responden que la evaluación "es algo que sirve para calificarnos..." (A3), o "para saber el nivel que tenemos" (A5). Este rasgo se confirma una vez más en los registros de la observación realizada, donde todos los comentarios docentes se realizan en base a conductas y cualidades propias de los alumnos.

Entendiendo la evaluación educativa de esta forma, se dejan a un lado varias de las características que la misma debería poseer, y que se mencionan tanto en la legislación como en el proyecto educativo del instituto. Al decir de Santos Guerra (2014), cuando esto sucede se agrega a la evaluación un componente jerárquico, ya que al ser los estudiantes los únicos evaluados, el poder sería manejado por los docentes y los alumnos deberían acatarlo, aceptando los resultados como su propia y única responsabilidad. Esto hace que la evaluación pase a ser en parte irracional, ya que desconoce otros factores que influyen en el proceso y en los resultados, y también injusta, haciendo al alumnado completamente responsable de sus logros y fracasos. Lo anterior llevaría a la omisión de la revisión y reflexión sobre las prácticas docentes, por lo cual el carácter formativo de la evaluación educativa se vería debilitado si concebimos que el mismo implica también la interpretación, el análisis y la reflexión sobre los esquemas y las prácticas de quien enseña (De Jesús, 2016; Hortigüela, Pérez-Pueyo y González-Calvo, 2019).

A su vez, son numerosos los autores que explican que la evaluación constituye una actividad crítica de aprendizaje, que se materializa en la recolección de información útil y veraz, en vías de mejorar la práctica educativa (Alcaraz, 2015b; Álvarez Méndez, 2008; Pérez Gómez, Soto Gómez, Sola y Serván Núñez, 2009; Santos Guerra, 2014). Santos guerra $(1993,1998$, cit. en Santos Guerra, 2014) agrega que esta ha de ser un proceso de diálogo entre evaluados y evaluadores, ya que cuanto mayor sea la participación de los evaluados en la evaluación, más formativa será esta última. Si retornamos ahora una vez más a las definiciones brindadas por los agentes educativos, encontramos que la que más se asemeja a esta concepción es la brindada por la orientadora, quien menciona que el objetivo de la evaluación es colaborar, e intentar mejorar para brindar la respuesta educativa más adecuada a cada estudiante. 


\section{E S T U D I A N T E S}

En cuanto a las definiciones de los docentes, hay diferencias entre quienes ponen el foco en los resultados (P1, P2, P3) y quienes hacen alusión al proceso de los estudiantes (P4, P5). Sin embargo, ninguno hace referencia al fin último de la evaluación mencionado por los autores consultados, ninguno parece perseguir prioritariamente la mejora de las prácticas educativas, sino más bien la comprobación de lo que ha aprendido el alumnado, lo cual según Alcaraz (2015a) responde a la confusión aún existente entre las características de la evaluación y de la calificación o lo que es igual, entre la evaluación formativa y la sumativa (Bigs, 2010, cit. en Ruay, 2018). Esta confusión no es menor, dado que si se materializa en las prácticas de enseñanza, la tarea más importante para el profesorado puede llegar a ser la determinación de situaciones en las que "el alumnado manifieste las conductas que reflejen la consecución o no, de los objetivos previstos" (Alcaraz, 2014, p.68). Lo cual terminaría por poner en peligro las cualidades de la evaluación propiamente educativa, haciendo que la misma ya no sea ni tan formativa, ni tan continua ni tan integradora, sino más bien certificadora.

... yo entiendo que la evaluación debe de ser educativa, y eso no es una... no
es solamente un adjetivo calificativo... yo creo que el objetivo de la evaluación
es colaborar e intentar mejorar. Cuando se evalúa creo que hay que decirle al
estudiante por donde debe seguir para seguir mejorando... Entonces evaluar
para calificar no... (...) Hay niños que necesitan más tiempo, otro que necesita
más contenidos de esto, otro que necesita más habilidades sociales... Entonces
la evaluación consiste en saber dónde hay que mejorar para proporcionar la
respuesta educativa que necesita cada estudiante en cada momento.

Tabla 3: ¿Qué significa evaluar? Respuestas de los docentes y la orientadora

Pasando ahora a las funciones de la evaluación, desde el proyecto de centro se enfatiza en la importancia de la continuidad y del carácter formativo de la misma, en vías de detectar y analizar las dificultades que aparezcan y poder mejorar el proceso de enseñanza-aprendizaje (PC, 2018). Esto es consonante con lo planteado por el decreto 111/2016 y la orden del 14 de julio de 2016, donde se asegura que; 


\section{E S T U D I A N T E S}

La evaluación se convierte, en sí misma, en un proceso educativo que considera al alumnado como centro y protagonista (...), que contribuye a estimular su interés y compromiso con el estudio, lo ayuda a avanzar en el proceso de asunción de responsabilidades y en el esfuerzo personal, y que le facilita el despliegue de sus potencialidades y su concreción de competencias necesarias para el desarrollo individual e integración social (p. 108).

Lo paradójico es que en la misma orden del 14 de julio de 2016, también se menciona que al finalizar cada curso y como consecuencia del proceso de evaluación, los equipos docentes deberán adoptar decisiones sobre la promoción del alumnado, atendiendo al logro de los objetivos y el grado de adquisición de las competencias correspondientes. Resulta de esta forma por lo menos confuso que se mezclen funciones de la evaluación, que por un lado se alinean a un paradigma constructivista del aprendizaje, pero por otro arrojan como consecuencia una acción de tipo conductista -como es certificar los conocimientos de los estudiantes para permitirles continuar en el sistema educativo-.

En cuanto a las opiniones de los docentes respecto a este tema, algunos argumentan que la evaluación sirve para comprobar lo que el alumno aprende, así como para medir su nivel (P1, P3), mientras otros destacan que debería servir como motivación para que los estudiantes tomen consciencia de su proceso y saber cómo ayudarlos (P4, P5). El problema según estos últimos, es que en la práctica diaria la evaluación termina sirviendo para promocionar, es decir, para poner una nota que certifique el pasaje de un curso a otro. Tomando en cuenta esto, puede observarse una vez más, como las opiniones difieren en cuanto a los fines que perseguiría la evaluación, pero los mismos recaen en todos los casos sobre el alumnado. A su vez, parece ser que se presenta en la mayoría de los docentes una dualidad, ya que por un lado resultan ser conscientes de la importancia de la evaluación para ayudar a los estudiantes, pero por otro, argumentan que en la vida de los hechos, la práctica camina en otra dirección. Lamentablemente, dicha dirección es la que busca, tal como ellos lo expresan: comprobar, medir y promocionar. Todo indicaría que se cumplen primero las funciones sociales, que dotan a la evaluación de un carácter de selectividad, clasificador y de control y luego, si queda tiempo o lugar, se recogerían las funciones pedagógicas, estrechadas con acepciones de orientación, motivación, retroalimentación y consolidación de los aprendizajes (De Jesús, 2016). Esto revelaría que la evaluación estaría siendo utilizada prioritariamente con fines académicos (Hortigüela, Pérez-Pueyo y González-Calvo, 2019), un error que puede llevar a pensar a los alumnos que el conocimiento posee un valor de cambio más que de uso (Fernández, 2015).

Los indicios anteriores parecen corroborarse en lo observado en la sesión de evaluación, ya que según el guion de desarrollo de la misma, se deberían: “(...) analizar las propuestas realizadas en la sesión anterior y llegar a conclusiones para generar nuevas propuestas para tenerlas en cuenta para el siguiente trimestre/curso" (GSE, 2018, 2019, p.2). Sin embargo, durante la observación realizada en ningún momento se mencionan las propuestas realizadas anteriormente, como base para la 


\section{E S T U D I A N T E S}

planificación y desarrollo de nuevas medidas. En cambio, si existen intercambios que ponen en el foco las conductas de los estudiantes y su relación con las calificaciones que estos últimos obtienen. Está a la vista una vez más, la confusión existente entre las funciones de la evaluación y de la calificación y la misma se trasmite a los alumnos, quienes al ser consultados sobre la utilidad de la primera, responden lo siguiente:

\begin{tabular}{ll}
\hline A2 & Para pasar al próximo curso, digo yo... \\
\hline A5 & Para saber el nivel que tenemos... \\
\hline A6 & $\begin{array}{l}\text { Pues para ver cómo ha ido durante el año, los exámenes que has hecho y todo } \\
\text { eso... }\end{array}$ \\
\hline A11 & Para hacernos sufrir \\
\hline A12 & Para saber las notas... para pasar de año. \\
\hline & Tabla 4: Respuestas del alumnado sobre las funciones de la evaluación \\
\hline
\end{tabular}

Puede observarse como las funciones que exponen dichos estudiantes concuerdan en gran medida con las mencionadas por los docentes. El propósito social de la evaluación vuelve a aparecer en primer lugar, habiendo una única alumna que hace alusión al proceso, tal como lo hicieron dos de los profesores entrevistados. El resto ha puesto el foco en los resultados, habiendo a su vez una respuesta que llama la atención, y me refiero a la otorgada por el alumno A11. Al decir del mismo, la evaluación sirve "para hacerlos sufrir" y es que cuando el énfasis de su práctica se pone en la comprobación, las pruebas pueden llegar a convertirse en una carrera de obstáculos, que los estudiantes deber sortear de la mejor manera posible para alcanzar el éxito en el sistema (Alcaraz, 2015a), o dicho de otra forma, para no ser excluidos del mismo. Se hace evidente que lo que los docentes creen sobre la evaluación y sus finalidades se refleja en sus prácticas y repercute inevitablemente en los estudiantes, condicionando en gran medida la definición que estos construirán sobre el mismo concepto. Está claro que para la mayoría de ellos, el fin último de la evaluación parece ser la certificación y lo importante parece ser lo que ya ha pasado, lo que se ha logrado o no.

Ahora bien, ¿cuál es el nivel de dificultad que perciben los docentes a la hora de poner en práctica la evaluación? Ante esta pregunta, la mayoría ha respondido que la evaluación no reportaría grandes dificultades. Únicamente una, particularmente la más joven, ha manifestado que sí, argumentando lo siguiente:

...evaluar a mi es casi que lo que me resulta más complejo porque siempre hay una parte que es más subjetiva, y más en las asignaturas de corte humanístico, cuando tienes que evaluar una impresión, una forma de redactar, un contenido... siempre hay una parte de subjetividad. Lo que pasa es que claro... siempre intentas aplicar el mismo parámetro para todos para que sea lo más objetivo posible. Pero es verdad que el evaluar a mi es lo que me resulta más complejo, porque al final lo que pones es una calificación que es un número y no creo yo que con un número se muestre que es lo que sabe o no sabe, o lo que ha aprendido o no ha aprendido un alumno... (P1) 
En su opinión pueden identificarse buenas intenciones, pero a la vez palparse una confusión de términos que hace que las primeras seguramente, no lleguen a concretarse. Ella habla de ser justa mediante la evaluación, pero cree que para poder serlo debe adoptar una postura objetiva y juzgar a todos los estudiantes mediante el mismo parámetro, y es allí donde puede radicar el mayor error. Ofrecer a todos lo mismo puede convertir a la evaluación en un mecanismo favorecedor de desigualdad (Santos Guerra, 2014), ya que si no se tienen en cuenta los diferentes puntos de partida de los estudiantes para actuar en consecuencia, las diferencias sociales no hacen más que acrecentarse. A su vez, la docente relaciona la complejidad de la evaluación con el componente de subjetividad, como si este último reportara una especie de problema y es que la subjetividad, efectivamente se convierte en un problema cuando la intención es calificar más que evaluar. A ello se agrega la inexistencia de triangulación entre lo que la docente cree y lo que consideran el resto de los agentes implicados en el proceso de enseñanza aprendizaje, lo cual condiciona ampliamente su pretendida objetividad.

Con respecto a esto último, la orientadora del instituto sostiene lo siguiente:

Yo, veo que algunas veces eh...el profesorado entiende la evaluación con un sentido de justicia, con lo cual quieren tratar a todos igual, a todos darles las mismas oportunidades... y eso a veces es engañoso (...) porque no todos tienen las mismas oportunidades, porque todos nacen y vienen de puntos de partida distintos. Quiero decir, que hay alumnos que lo tienen muy bien (...) y hay niños y niñas que tienen un contexto más deficitario, y el profesorado, creo que, no sé... tenemos que tender a compensar a aquellos que tienen peores situaciones de partida. No quiero decir ponérselo más fácil... (...) pero si tener en cuenta que las situaciones de partida no son las mismas, que hay que compensar (...) para que puedan llegar al mismo sitio... pero para eso está justamente el sistema educativo... (E0).

Parecería ser que muchas veces resulta complejo de comprender para el profesorado, que la igualdad de oportunidades no radica en ofrecer a todos lo mismo, sino en ajustar las prácticas educativas a las características de los estudiantes, en vías de compensar las desigualdades existentes. Si esto no se tiene en cuenta y la evaluación, con aparente igualitarismo, trata a todos por igual, no hace más que perjudicar a quienes la sociedad ya ha perjudicado de partida (Anyon, 1983, cit. en Fernández, Alcaraz y Sola, 2017; Apple, 1991, cit. en Fernández, Alcaraz y Sola, 2017; Bourdieu y Passeron, 1981, cit. en Fernández, Alcaraz y Sola, 2017; Santos Guerra, 2014). Y es que existe una estrecha relación entre rendimiento académico y nivel sociocultural (Organización para la Cooperación y el Desarrollo Económico, 2012) que si se omite sin más lleva a una inexorable pérdida de equidad; esa equidad y calidad de la educación proclamada por la LOMCE, la cual expone precisamente que "no es imaginable un sistema educativo de calidad en el que no sea una prioridad eliminar cualquier atisbo de desigualdad" (Ley Orgánica 8/2013, de 9 de diciembre, para la mejora de la calidad educativa, p. 97860).

Por otra parte, si bien, tal como se dijo anteriormente, la mayoría de los docentes entrevistados argumentan que la evaluación no les resulta complicada, en la sesión 


\section{E S T U D I A N T E S}

de evaluación observada se evidencia como los estudiantes arrastran los suspensos, como si fueran deudas que acumulan de trimestre en trimestre. Si concebimos a la evaluación como esa actividad crítica de aprendizaje, que recoge información de forma sistemática para mejorar las prácticas, ¿por qué no se reflejan esas mejoras en los procesos del alumnado? Quizás esto suceda porque como dice Álvarez Méndez (2008) en las instituciones se examina mucho y se evalúa poco, o al decir de Santos Guerra (2014), se evalúa mucho y se cambia poco. Y ello indicaría que algo está fallando, ya que si como manifiesta Stenhouse (1984) evaluar es comprender, deberían desligarse cambios a partir de dicha comprensión. Quiero decir, que mediante la evaluación deberían extraerse consecuencias para el debate y la mejora, pero sin embargo, las sesiones de evaluación están más bien enfocadas en lo que hace o no hace el estudiante, y no en lo que se puede modificar en el currículum a partir de la comprensión de su comportamiento (Santos Guerra, 2014).

\subsection{El procedimiento de evaluación}

Respecto a dicha categoría, resulta de importancia destacar que más allá de las perspectivas docentes, que influyen inevitablemente en las prácticas de evaluación que se llevan a cabo dentro del aula, estas últimas también se encuentran determinadas por otros factores como la formación, las condiciones laborales, las exigencias contradictorias de las leyes (que ya han sido mencionadas y se continuarán analizando), el tiempo, y las exigencias del currículum (Alcaraz, 2015b; Santos Guerra, 2014). Es evidente y no podemos olvidar que todos los elementos anteriores condicionan en gran medida el proceso de evaluación.

Habiendo aclarado lo anterior, comenzaremos a analizar la transparencia de los criterios de calificación y evaluación expuesta por los docentes del curso. En principio, parece trascendente mencionar que tanto la orden de 14 de julio de 2016, como el decreto 111/2016, especifican que los centros deben hacer públicos los criterios de evaluación y promoción y que es un derecho propio del alumnado el conocimiento de estos últimos. Además, son diversos los autores que afirman que los criterios de valoración y de corrección deben ser clarificados y conocidos por los estudiantes, para hacer de la evaluación un ejercicio transparente (Álvarez Méndez, 2008; Arredondo, 1999, cit. en De Jesús, 2016; Hortigüela, Pérez-Pueyo y González-Calvo, 2019, Pasek y Mejía, 2017; Pérez Gómez, Soto Gómez, Sola y Serván, 2009; Ruay, 2018). La mayoría de los docentes consultados afirman que efectivamente lo hacen, pero en sus argumentos explican que esto se hace necesario para que el alumnado comprenda el porqué de las notas, o de los puntos que vale cada actividad. Estos comentarios dan cuenta clara de la exposición de criterios de calificación, pero no precisamente de evaluación.

Evidentemente, cuando poner calificaciones es algo inevitable, resulta un factor clave que los estudiantes conozcan los criterios que se utilizarán para su asignación (Ruay, 2018). Que las notas no sean puestas “a ojo” y el alumnado tenga claro cómo será valorado su desempeño (Hortigüela, Pérez-Pueyo y González-Calvo, 2019; Pa- 


\section{E S T U D I A N T E S}

sek y Mejía, 2017), suele brindar seguridad y disminuir en parte, esa ansiedad que genera el tan nombrado sistema de calificaciones. Pero los criterios de evaluación no persiguen dicho objetivo, sino que implican el compartir con los estudiantes las razones por las cuales se realizan las tareas, los motivos en los que se asientan las mismas y la utilidad que poseen (Pérez Gómez, Soto Gómez, Sola y Serván, 2009). Esto es lo que llevará al alumnado a desarrollar una mayor autonomía, a poder autoevaluarse y a regular su propio aprendizaje (Arredondo, 1999, cit. en De Jesús, 2016). Por ello resulta tan importante invertir tiempo en debatir al respecto, y lograr que se genere una auténtica comprensión de estos últimos factores.

Los estudiantes, al ser consultados sobre este punto, mencionan en sus respuestas que los docentes les aclaran cuales son las actividades que valen más y menos puntos, o el tipo de pruebas que tendrán que cumplir a lo largo del año. Esto parece indicar que se dedica un tiempo mayor a explicar y reflexionar sobre el porqué de las calificaciones, que sobre el porqué de los procesos que tienen lugar en el aula. Una vez más, parece priorizarse el valor de cambio, sobre el valor de uso del conocimiento (Santos Guerra, 2014).

En cuanto a los instrumentos que se utilizan para la evaluación, el decreto 327/2010, de 13 de julio, por el que se aprueba el Reglamento Orgánico de los Institutos de Educación Secundaria, otorga autonomía a los docentes para emplear los métodos de enseñanza y aprendizaje que consideren más apropiados de acuerdo al nivel de desarrollo, aptitudes y capacidades del alumnado, en consonancia con lo establecido en el proyecto educativo del instituto (artículo 10, punto 2.b.). En lo que respecta precisamente a los métodos de evaluación, el proyecto educativo del instituto hace alusión a los mismos únicamente al referirse a la evaluación inicial, afirmándose que el profesorado utilizará los que considere más adecuados afín de conocer y valorar la situación de los estudiantes (PC, 2018). Parecería por lo tanto existir cierta libertad de elección para los docentes al momento de seleccionar los instrumentos de evaluación, aunque dicha libertad se ve condicionada teniendo en cuenta que luego estos deberán justificar la asignación de una calificación determinada -basada en la comprobación del alcance de los objetivos establecidos para la etapa-, a la vez que promover de manera paulatina "la autogestión del esfuerzo personal y el autocontrol del alumnado sobre el propio proceso de aprendizaje" (Orden de 14 de julio de 2016, p. 108), dos funciones que sin dudas, parecen difíciles de compatibilizar.

Los docentes de este curso hacen alusión a diversas herramientas de evaluación, pero en todas sus respuestas hay una que se repite una y otra vez: los exámenes. Esto es algo que corrobora la orientadora, quien afirma que el profesorado utiliza mucho este tipo de pruebas, y que las complementa con otras como los ejercicios de clase y la observación. A su vez, en las respuestas de los estudiantes se confirma dicha tendencia, ya que todos mencionan las pruebas escritas, mientras algunos agregan las tareas en clase y en casa, así como el comportamiento. En la sesión de evaluación observada, la importancia otorgada al examen se manifiesta en comentarios como el siguiente; 
(...) "en mi clase intenta hacer los ejercicios y a veces cuando trabaja en grupo logra buenos resultados, pero en los exámenes le va muy mal. Mi asignatura la tiene suspensa y no creo que mejore demasiado."(RO)

Algunos docentes dicen ofrecerle más y otros menos "peso", algunos parecen confiar en que dicho instrumento ofrece información válida e importante, mientras otros opinan que son pocos los datos que brinda sobre el aprendizaje de los estudiantes, pero todos lo utilizan en sus prácticas de evaluación. Con respecto al mismo, Álvarez Méndez (2001, cit. en Alcaraz, 2015b) señala que en el momento de su realización ya no hay lugar para la duda ni el error, los malentendidos o las distracciones y de esta forma, se reduce el aprendizaje a la capacidad de repetición y retención, más que a la elaboración de pensamiento propio. Además, en su esencia y por definición, el énfasis de dicho método está puesto en la reproducción. Así, en muchos casos se termina por reducir el aprendizaje a la memorización, haciéndose difícil encontrar explicaciones causales a los resultados obtenidos por los estudiantes (Álvarez Méndez, 2008; Santos Guerra, 2014). Fernández, Alcaraz y Sola (2017) agregan además que los resultados que estas pruebas pueden evidenciar, no equivalen al aprendizaje real, ya que este no puede ser medido, al tratarse de modificaciones en las estructuras cognitivas de quienes aprenden. Parecería ser entonces que este instrumento por sí solo no puede dar cuenta del grado de adquisición de las competencias que se pretenden desarrollar en el alumnado, y mucho menos, explicar los aciertos y las dificultades que este último encuentra en el proceso de enseñanza aprendizaje. De hecho, Pérez Gómez, Pérez Soto, Sola y Serván (2009) afirman que la mayoría de los exámenes requiere sólo competencias de nivel inferior, ignorando otras de nivel superior como el análisis, la síntesis o la reflexión. Todo lo cual nos puede llevar a pensar que dicha herramienta es más bien útil para cumplir con la justificación de la calificación, pero no para promover el autocontrol y la autogestión de los estudiantes.

Con respecto a los demás instrumentos de evaluación que aparecen también en varias respuestas, encontramos las tareas diarias y la observación, que da lugar a la ponderación de variables como el esfuerzo, el compromiso y la actitud del alumnado. Respecto a las primeras, no se cuenta con información suficiente como para juzgar los objetivos a los cuales aspiran, ni las características específicas que poseen. En relación a la observación, que parece ser determinante a la hora de establecer juicios sobre el comportamiento así como sobre las actitudes del alumnado, Álvarez Méndez (2008) afirma que la misma suele ser utilizada por los docentes de forma espontánea e intuitiva. Lo que no queda del todo claro en este caso es, en principio, si el profesorado documenta este tipo de evaluación -mediante diarios, anotaciones, registros- y luego, si la acompaña de instancias de diálogo con los estudiantes, ya que este último permitiría valorar con mayor profundidad los motivos en los que se asienta su comportamiento (Álvarez Méndez, 2008; Pasek y Mejía, 2017; Santos Guerra, 2014).

En definitiva, si nos basamos en lo dicho por los docentes así como por los estudiantes, en este caso estaría presente la triangulación de métodos o estrategias 


\section{E S T U D I A N T E S}

de evaluación, pero no así la de sujetos, ya que no parecería dársele el lugar al alumnado para hacer valer su palabra y argumento, así como tampoco se menciona la participación de las familias en el proceso. Quedan dudas por lo tanto sobre si el uso de los instrumentos se alinea con una visión de la evaluación como proceso que promueve el aprendizaje, o más bien como un control externo del profesorado sobre el alumnado (Perrenoud, 2008, cit. en Ruay, 2018). No debemos olvidar que para que la evaluación se considere educativa, las voces de todos los agentes educativos deben ser escuchadas, en vías de alcanzar una mayor comprensión del proceso (Alcaraz, 2015b).

En consonancia con esto último y con respecto al feedback sobre el proceso, a pesar de que tanto el PC (2018) como el guion para el desarrollo de la sesión de evaluación $(2018,2019)$ mencionan la posibilidad de que los delegados del curso dialoguen con los docentes, pudiendo exponer diversas cuestiones que afecten al grupo, esto no se sostiene en la práctica del instituto. De hecho, en la sesión de evaluación observada no hubo presencia de estudiantes, ni tampoco se hizo alusión a sus opiniones por parte de los tutores. Ahora bien, podría deducirse que si esta práctica se materializara, y se elaborara con reflexión y detenimiento, de manera sistemática, los alumnos podrían brindar datos sinceros y relevantes para la mejora de las prácticas de enseñanza (Alcaraz, 2015b; Álvarez Méndez, 2008). Así, la evaluación sería sin dudas más democrática, formativa y motivadora.

En cuanto a la retroalimentación de los docentes a los estudiantes, la misma resulta un factor fundamental si se concibe a la evaluación educativa tal como se ha especificado en las leyes y en el proyecto educativo de centro, es decir, como una de carácter continuo y formativo, que aspira a la mejora. Si se adopta dicha perspectiva, no se puede olvidar que la evaluación siempre debe estar al servicio de los protagonistas, y sobre todo, de los sujetos que aprenden (Álvarez Méndez, 2008). Las devoluciones formarían parte del quehacer del profesorado en cuanto a este último le corresponde orientar y apoyar al alumnado en su proceso educativo (Decreto 327/2010, de 13 de julio de 2010).

Extrapolando lo anterior al instituto en cuestión, según la orientadora, los docentes parecerían cumplir con dicha labor ya que "se va dando al alumno constantemente retroalimentación de qué tiene que hacer para mejorar" (EO). El profesorado por su parte, admite que intenta ofrecer devoluciones al alumnado. De hecho, la primera docente entrevistada resalta el efecto motivador que las mismas reportan. El hecho es que no cualquier tipo de devolución, hecha en cualquier momento del proceso tiene el mismo valor, ni llega a los alumnos de la misma forma. Los comentarios realizados después de los exámenes tienen un carácter más bien retroactivo (De Jesús, 2016), debido a que se ofrecen cuando ya no hay tiempo para reflexionar y mejorar. Este tipo de feedback no colaboraría en gran medida con la autorregulación, autonomía y metacognición de los estudiantes (Hortigüela, Pérez-Pueyo y González-Calvo, 2019). A su vez, resulta de gran importancia que la información brindada sea comprensible y esclarecedora para el alumnado, ya 


\section{E S T U D I A N T E S}

que como plantea Álvarez Méndez (2008), la clave del feedback se encuentra en la calidad y claridad de los datos y a través del mismo, el docente debe contribuir a encontrar soluciones a tiempo, que eviten el fracaso y apuesten por la superación.

Asimismo, algunos docentes mencionan que el objetivo de las devoluciones es que los alumnos puedan mejorar mediante la detección de lo que han hecho mal. Otra vez, el foco se pone en estos últimos; si cometen errores, parecen ser ellos los únicos responsables. Igualmente, podríamos decir que al aspirar a la reflexión sobre el proceso, el profesorado poseería buenas intenciones. Ahora bien, estas deben acompañarse de otros elementos para lograr ser enriquecedoras, como por ejemplo, un clima de confianza mediante el cual se promueva la autoevaluación, y el error no sea penalizado sino comprendido y transformado (Alcaraz, 2015b, Hattie, 2017).

En definitiva, si nos apoyáramos en las respuestas de los docentes podríamos decir que se ofrecen fundamentalmente dos tipos de feedback; uno más bien orientado a la tarea, y otro, más dirigido al proceso. El hecho es que según las respuestas aportadas por los estudiantes, parece ser que el que ellos más identifican es el que se corresponde con la tarea, aquel mediante el cual el profesorado le comunica si la ha hecho bien o mal, pero no mucho más que eso. El alumnado no presenta ejemplos de comentarios docentes que se relacionen con la reflexión sobre el proceso, lo cual puede deberse a que les cueste identificarlos explícitamente, o quizás, a que no terminan de apropiarse de la información por diversos motivos. Entre estos puede estar el hecho de que el feedback sea brindado a toda la clase en conjunto, resultando un tanto confuso e incomprensible (Hattie, 2017), o que los comentarios otorgados sean demasiado breves (Pérez Gómez, Soto Gómez, Sola y Serván, 2009) y no establezcan orientaciones específicas que conecten con el lugar del proceso en que se encuentran los estudiantes.

Lo preocupante son las veces que se repiten las frases de tipo "tienes que estudiar más", "te tienes que esforzar" o "tienes que trabajar más en casa", ya que todas ellas dan cuenta de procesos atributorios exculpatorios (Santos Guerra, 2014) que ponen toda la responsabilidad de los malos resultados sobre los estudiantes, ignorándose otras posibles variables que pueden influir en la díada de enseñanza-aprendizaje. Además, son evidentes las posibles repercusiones que este tipo de devoluciones sumadas a las calificaciones pueden generar en los estudiantes. Entre ellas, encontramos la propulsión de comportamientos perversos que van en dirección opuesta al aprendizaje, a los cuales Rodríguez (2003, cit. en, Alcaraz, 2015b) denomina como efectos de la racionalidad calificativa. "Hacer la pelota", tal como dice el alumno A11, copiar, quedar bien, mentir, son algunas de las conductas que entrarían dentro de este conjunto de comportamientos y que evidentemente, nada tienen que ver con los objetivos que persigue la evaluación educativa. 


\section{E S T U D I A N T E S}

\subsection{La calificación}

A través de esta última categoría, dividida en dos subcategorías específicas, se pretende comprender el concepto que los docentes y alumnos manejan sobre la calificación, así como el reparto del peso que se realiza de esta última.

Es de público conocimiento que en el sistema educativo, dada su estructura y condiciones, la evaluación y la calificación deben convivir, lo cual genera muchas veces confusión entre las mismas y sus funciones. Inclusive a nivel legislativo, en decretos y órdenes estas confusiones se hacen patentes, por ejemplo, cuando se hace alusión a la promoción del alumnado y se indica que este último no podrá promocionar cuando su evaluación en tres materias o más haya sido negativa. Evidentemente, estos son aspectos que no colaboran con la diferenciación entre los términos. Respecto a ello, la orientadora del instituto plantea lo siguiente;

Creo que la tendencia de nuestro sistema educativo es a confundir, evaluación con calificación, pero que luego cuando hablamos del concepto de evaluación educativa, esa evaluación que necesitas para mejorar, todo el mundo sabe de lo que estamos hablando y se pone en marcha. (EO)

Parecería ser entonces que aunque se admite la tendencia a confundir ambos conceptos, los mismos serían diferenciados al momento de ponerlos en práctica y efectivamente, a partir de las respuestas docentes, se desligan indicios de distinción entre los mismos. Parece comprenderse que la evaluación es más compleja, que implica otras acciones y que debería servir para ayudar a los estudiantes. El problema es que lo que diferencia a la calificación y a la evaluación va mucho más allá de eso; la calificación, más que ir a favor, obstruye el proceso de enseñanza aprendizaje, vinculándolo inevitablemente con una pedagogía transmisiva que se sabe ya como obsoleta e ineficaz (Feito, 2010, cit. en Alcaraz, 2015c).

Uno de los docentes entrevistados argumenta que no le encuentra sentido a la calificación, pero a su vez, admite que a los alumnos que no logran comprometerse y que él no logra acercar a la asignatura, no tiene otra alternativa que dejarlos suspensos. Se hace evidente así la función sancionadora de la calificación, su sentido de control y acreditación social (Pérez Gómez, Soto Gómez, Sola y Serván, 2009). ¿Qué sucedería si en vez recurrir a la calificación, se la eliminara y se centraran los esfuerzos en indagar lo que le sucede a los estudiantes?, ¿qué ocurriría si en vez de asumir la autoridad mediante el sistema de calificación, el profesorado generara una relación horizontal que permitiera una mayor confianza para poder dialogar con sinceridad? Quizás este cambio de perspectiva arrojaría mayores beneficios sobre el aprendizaje. Ahora bien, volviendo a lo que ocurre actualmente, está claro que la calificación no se preocupa por las causas o procesos que han llevado a los aparentes resultados, sino que se conforma con medir el conocimiento adquirido y en función de ello clasificar, certificar, lo cual es algo peligroso, y más, si tomamos en cuenta el contexto en el cual está inmerso el instituto. 
Lo malo es que, aun siendo cierto el peligro mencionado, parece ser que el número de suspensos y aprobados que arroja la calificación ocupa un lugar significativo en la agenda de los docentes y del centro educativo, lo cual se ve reflejado también en la sesión de evaluación observada donde al plantearse la situación de cada alumno en particular, la secuencia siempre era la misma; se mencionaba primero su nombre, luego el número de suspensos y finalmente los comentarios al respecto de su comportamiento, actitud, participación en clase, etcétera.

Dicha relevancia otorgada a la calificación se hace patente en las respuestas aportadas por los estudiantes, ya que varios creen que la evaluación es precisamente el número final, es decir, la media entre todas las notas obtenidas a lo largo del curso, mientras la calificación se correspondería con las puntuaciones parciales. La importancia dada a esta última podría ser en parte la responsable de la confusión de los estudiantes, quienes en definitiva consideran que el objetivo de la evaluación sería arribar a un número, que más que informar sobre el proceso ejerce una función de control sobre ellos mismos.

Ahora bien, ¿cómo se reparte el peso de la calificación? De acuerdo a los indicios que aporta el profesorado, lo que más importancia tendría para el establecimiento de la calificación sería; la observación en clase -en vías de detectar el esfuerzo, compromiso, participación y trabajo diario de los estudiantes- y los resultados de los exámenes. Podríamos pensar que la elección de dichas fuentes o técnicas se basa en la creencia de que aportan información fiel sobre los aprendizajes "adquiridos", lo cual responde a la evidente y confusa relación entre la calificación y la evaluación. Ahora bien, también es cierto que existe cierta coerción por parte del sistema hacia los docentes (Santos Guerra, 2014), y que la misma podría condicionar esta decisión. Me refiero concretamente a que, más allá de la valoración del trabajo diario, el sistema educativo pide "evidencias objetivas" que respalden la medición llevada a cabo y esto se traduce casi indefectiblemente, en exámenes. Lo paradójico es que en el afán de recopilar esas evidencias, el profesorado cede ante la realización de estos últimos (aunque parezca no estar de acuerdo), pero a la par de ello, recurre también a una observación que parece ser a todas luces no sistemática, ni registrada, ni triangulada con otras fuentes de información. Esto resulta contradictorio si pensamos en la pretendida objetividad anteriormente mencionada, ya que finalmente parecería que la calificación y por ende la clasificación del alumnado se sustentaría, por un lado en una visión que puede tener mucho de intuición y poco de sistematización, y por otro en la utilización de un instrumento que más que interpretar al aprendizaje como construcción, lo concibe como una reproducción.

Recogiendo ahora lo expresado por los estudiantes, los mismos notan y reconocen la importancia de los exámenes así como de la observación, y son conscientes de su peso en la calificación. En sus respuestas admiten además la valoración de los trabajos domiciliarios y de aula, algo que también sostuvo el profesorado, pero parecería ser que estos se tienen en cuenta en menor medida que los anteriores. 


\section{E S T U D I A N T E S}

\section{CONCLUSIONES}

Mediante la realización de este estudio, se ha intentado conocer y comprender las opiniones de docentes y alumnos de $1^{\circ}$ de la ESO de un instituto al respecto de la evaluación educativa; su concepción, sus funciones, su procedimiento materializado en las prácticas y su diferenciación respecto de la calificación.

Tal como se ha afirmado a lo largo de dicha descripción, la línea del trabajo ha intentado seguir la lógica del estudio de caso, pero se ha aclarado también que no se ha cumplido en toda regla con esta metodología, ya que las condiciones y el tiempo han limitado el rigor del proceso. Existen diversos factores que podrían generar una aproximación más fiel, rigurosa y rica al caso en cuestión, y dentro de ellos podemos encontrar: una triangulación que se valiera de más fuentes de datos (la revisión de trabajos de los estudiantes, los cuestionarios que no pudieron ser completados, observaciones dentro del aula), la revisión de los interesados (Stake, 2010) o reflexión de los miembros (Tracy, 2010) y también un involucramiento por un mayor periodo de tiempo (Maxwell, 2012) dentro del instituto.

En cuanto a los datos obtenidos sobre la realidad del curso e instituto que ha sido objeto de estudio, se puede apreciar que el concepto de evaluación educativa manejado por los docentes es en parte confuso, y se encuentra centrado en el alumnado. Las funciones parecen solaparse con las de la calificación, lo cual termina repercutiendo en el proceso que llevan a cabo los docentes para realizar la evaluación; en los instrumentos que utilizan, en los datos que recopilan, en la información que ofrecen al alumnado, y también en como este último la recibe. La calificación, parece tener un gran peso para ambos agentes educativos y junto a las características del sistema educativo en general, del instituto en particular, y las creencias de los educadores, condicionan ampliamente la práctica evaluadora. Parece ser que las intenciones de estos últimos son muchas veces adecuadas pero el tiempo, la cantidad de alumnos, la formación y las exigencias desde los niveles más altos del sistema son factores que no suelen jugar a favor.

Con respecto a los cambios que se necesitarían para modificar la concepción de la evaluación y sus prácticas, Perrenoud (2008, cit. en Pereira, 2015) argumenta que sería imprescindible la reconstrucción de las instituciones educativas, pues la evaluación está en el núcleo del sistema didáctico y de enseñanza. En consonancia con ello, Álvarez Méndez (2008) agrega que no es posible cambiar un elemento de la estructura sin alterarla por completo. Hace falta cambiar el sistema, desde la formación que se ofrece a los docentes hasta la educación de los adolescentes y niños. Ahora bien, mientras estos cambios no sean posibles y evaluación y calificación deban coexistir en los centros educativos, se pueden reestructurar ciertas prácticas, de forma de acercarlas a la función formativa que debería caracterizar a todo proceso evaluador. Es importante en este sentido, el uso que se haga de los instrumentos, así como el valor que se le otorgue a la información que los mismos arrojan. 


\section{E S T U D I A N T E S}

Es necesario que la evaluación sea transparente, creíble, con coherencia epistemológica y cohesión práctica, pertinente, aplicable y legítima, y para ello, debe estar al servicio de quien enseña y de quien aprende. Esto último constituye un componente fundamental que todos los agentes educativos deberían comprender, ya que conforma la base necesaria para poder cambiar la relación entre alumnado y profesorado, y que la misma pase a sustentarse en la cooperación, participación y corresponsabilidad.

Ahora bien, para que lo anterior tenga lugar, es necesario trabajar con docentes y con estudiantes. En principio, fundamentalmente con los primeros, para que puedan reflexionar y cuestionar el sentido del aprendizaje y la función que las instituciones educativas desempeñan y recién después, replantearse el papel de la evaluación. Esta última debe servir para establecer y negociar claramente los propósitos del proceso de aprendizaje, debe brindar una retroalimentación constructiva, desarrollar la co-evaluación y la auto-evaluación y por último, plantear tareas auténticas; contextualizadas y significativas para quienes aprenden (Pasek y Mejía, 2017; Pérez Gómez, Soto Gómez, Sola y Serván, 2009).

\section{REFERENCIAS BIBLIOGRÁFICAS}

Alcaraz, N. (2014). Un viejo trío de conceptos: Aprendizaje, currículum y evaluación. Aula de Encuentro, 16(2), 55-86.

Alcaraz, N. (2015a). Aproximación Histórica a la Evaluación Educativa: De la Generación de la Medición a la Generación Ecléctica. Revista Iberoamericana de Evaluación Educati$v a, 8(11), 11-25$.

Alcaraz, N. (2015b). La evaluación en el aula. Explorando el portafolios en Secundaria. Un estudio de caso. (Tesis de Doctorado). Universidad de Málaga, Málaga, España.

Alcaraz, N. (2015c). Evaluación versus Calificación. Aula de Encuentro, 17(2), 209-236.

Alfageme, B., Miralles, P. y Monteagudo, J. (2011). Diseño y validación de un instrumento sobre evaluación de la geografía y la historia en educación secundaria. Enseñanza de las ciencias sociales, 10, 51-63.

Álvarez Méndez, J.M. (1993). La evaluación como actividad crítica de aprendizaje. Cuadernos de Pedagogía, 219, 28-32.

Álvarez Méndez, J. M. (2004). La evaluación educativa al servicio de quien aprende: el compromiso necesario con la acción crítica. En La formación docente: Evaluaciones y nuevas prácticas. Santa Fe, Argentina: UNL, pp. 11-23.

Álvarez Méndez, J.M. (2008). Evaluar para conocer, examinar para excluir. Razones y propuestas educativas. Madrid: Morata.

Campos, G. y Lule, N. (2012). La observación, un método para el estudio de la realidad. Revista Xihmai, 7(13), 45-60.

Decreto 327/2010, de 13 de julio, por el que se aprueba el Reglamento Orgánico de los Institutos de Educación Secundaria. Boletín Oficial de la Junta de Andalucía, núm. 139, de 16 de julio de 2010, pp. 8 a 34. Recuperado de: https://www.juntadeandalucia.es/ boja/2010/139/d2.pdf

Decreto $111 / 2016$, de 14 de junio, por el que se establece la ordenación y el currículo de la Educación Secundaria Obligatoria en la Comunidad Autónoma de Andalucía. Boletín 
Oficial de la Junta de Andalucía, núm. 122, de 28 de junio de 2016, pp. 27 a 45 . Recuperado de: https://www.juntadeandalucia.es/boja/2016/122/B0JA16-122-0001911633-01_00094130.pdf

De Jesús, E. (2016). La función formativa de la evaluación en un currículum integral. (Tesis de Doctorado). Universidad Complutense de Madrid, Madrid, España.

Fernández Navas, M. (2015). Internet, organización en red y educ@ción. Estudio de un caso de buenas prácticas en enseñanza superior (Tesis Doctoral). Universidad de Málaga, Málaga, España.

Fernández Navas, M., Fernández Millán, J.M. y Hamido Mohamed, A. (2011). Educación social y atención a la infancia. Madrid: Pirámide.

Fernández Navas, M., Alcaraz, N. y Sola, M. (2017). Evaluación y pruebas estandarizadas: una reflexión sobre el sentido, utilidad y efectos de estas pruebas en el campo educativo. Revista Iberoamericana de Evaluación Educativa, 10(1), 51-67.

Flick, U. (2012). Introducción a la investigación cualitativa. Madrid: Morata

Flyvbjerg, B. (2006). Five Misunderstandings About Case-Study Research. Qualitative Inquiry, 12(2), 219-245.

Hattie, J. (2017). Aprendizaje visible para profesores. Maximizando el impacto en el aprendizaje. Madrid: Ediciones Parainfo.

Hortigüela, D., Pérez-Pueyo, A. y González-Calvo, G. (2019). Pero...¿A qué nos referimos realmente con la Evaluación Formativa y Compartida: Confusiones Habituales y Reflexiones Prácticas. Revista Iberoamericana de Evaluación Educativa, 12(1), 13-27.

Lagoa, L. (2014). Adolescentes Resilientes: Características en Estudio de Caso en Instituciones Educativas de Contexto Crítico de Montevideo. (Tesis de pregrado). Universidad Católica del Uruguay, Montevideo, Uruguay.

Ley Orgánica 8/2013, de 9 de diciembre, para la mejora de la calidad educativa. Boletín Oficial del Estado. Madrid, 10 de diciembre de 2013, núm. 295, pp. 97858 a 97921. Recuperado de: https://www.boe.es/buscar/pdf/2013/BOE-A-2013-12886-consolidado. pdf

Maxwell, J. A. (2012). Qualitative research design: An interactive approach. Londres: Sage Publications.

McMillan, J. y Schumacher, S. (2005). Investigación educativa. Una introducción conceptual (5ta ed.). Madrid: Pearson Educación.

Morey, A. (2001). La Evaluación Formativa en la Educación Secundaria Obligatoria. Educació i Cultura, 14, 183-200.

Muñoz, P. y Muñoz, I. (2000). Intervención en la familia: Estudio de casos. En G. Pérez (Coord.) Modelos de investigación cualitativa en educación social y animación sociocultural (221- 252). Madrid: Narcea Ediciones.

OCDE. (2012). Estudiantes de bajo rendimiento. Por qué se quedan atrás y cómo ayudarles a tener éxito. París: OECD Publishing. Recuperado de: http://www.oecd.org/pisa/keyfindings/PISA-2012-Estudiantes-de-bajo-rendimiento.pdf

Orden de 22 de julio de 1999, por la que se regulan las actuaciones de compensación educativa en centros docentes sostenidos con fondos públicos. Boletín Oficial del Estado, núm. 179, de 28 de julio de 1999, pp. 28052 a 28056. Recuperado de: https://www. boe.es/boe/dias/1999/07/28/pdfs/A28052-28056.pdf

Orden de 14 de julio de 2016, por la que se desarrolla el currículo correspondiente a la Educación Secundaria Obligatoria en la Comunidad Autónoma de Andalucía, se regulan determinados aspectos de la atención a la diversidad y se establece la ordenación de la evaluación del proceso de aprendizaje del alumnado. Boletín Oficial de 
la Junta de Andalucía, núm. 144, de 28 de julio de 2016, pp. 108 a 396. Recuperado de: https://www.juntadeandalucia.es/boja/2016/144/B0JA16-144-00289-1350001_00095875.pdf

Osses Bustingorry, S., Sánchez Tapia, I., y Ibáñez Mansilla, F. (2006). Investigación cualitativa en educación: hacia la integración de teoría a través del proceso analítico. Estudios pedagógicos (Valdivia), 32(1), 119-133. doi: https://dx.doi.org/10.4067/S071807052006000100007

Pasek, E. y Mejía, M. (2017). Proceso General para la Evaluación Formativa del Aprendizaje. Revista Iberoamericana de Evaluación educativa, 10(1), 177-193.

Pereira, J. (2015). Evaluación, medición o verificación de los aprendizajes en el aula: Un estudio de caso en el Colegio Humanístico Costarricense de Heredia. Revista Electrónica Educare, 19(2), 405-428. doi: http://dx.doi.org/10.15359/ree.19-2.22

Pérez Gómez, Á., Soto Gómez, E., Sola, M. y Serván Núñez, M. J. (2009). La evaluación como aprendizaje. ¿Evaluación = Calificación? Madrid: Akal.

Popham, W.J. (Coord.). (2013). Evaluación trans-formativa. El poder transformador de la evaluación formativa. Madrid: Narcea.

Ruay, R. (2018). La evaluación: una estrategia para desarrollar aprendizajes profundos en el estudiante. Boletín Redipe, 7(8), 46-52.

Rubio, J., Castillejo, J., Sánchez, J. y López, M. (1992). Acción tutorial en educación secundaria. En R. Bautista, I. Canca, J. Castillejo, M.L. González, M. López, E. de la Plata, J. Rubio, J.M. Sánchez y A. Varón. Orientación e Intervención Educativa en Secundaria (37-78). Málaga: Aljibe.

Santos Guerra, M. A. (2014). La Evaluación como Aprendizaje. Cuando la flecha impacta en la diana. Madrid: Narcea.

Schutt, R. (2011). Investigating the Social World: The Process and Practice of Research (7ma ed.). California: Sage Publications.

Stake, R.E. (2010). Investigación con estudio de casos (5ta ed.). Madrid: Morata.

Stenhouse, L. (1984). An introduction to curriculum research and development. Londres: Heinemann.

Tracy, S. (2010). Qualitative Quality; Eight “Big-Tent” Criteria for Excellent Qualitative Research. Qualitative Inquiry, 16, 837-851. 